Journal of Applied Fluid Mechanics, Vol. 13, No. 6, pp. 1847-1858, 2020.

Available online at www.jafmonline.net, ISSN 1735-3572, EISSN 1735-3645.

DOI: $10.47176 / \mathrm{jafm} .13 .06 .31238$

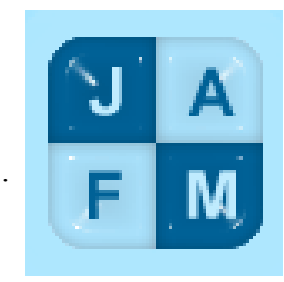

\title{
Numerical Analysis of Flow Geometry in I-Shaped Viscous Micropumps using LB-IBM
}

\author{
A. Alimoradi and S. Ali Mirbozorgi ${ }^{\dagger}$ \\ Department of Mechanical Engineering, University of Birjand, Birjand, Iran \\ †Corresponding Author Email: samirbozorgi@birjand.ac.ir
}

(Received December 10, 2019; accepted May 7, 2020)

\begin{abstract}
In viscous micropumps one of the main reasons for a flow rate reduction is vortices which are located at the top of the rotating rotor. In this paper, we have tried to add proper additional walls in the micropump channel, to eliminate or decrease the size of these vortices. Among the all investigated new models, only one, the I-Shaped micropump with an extra step above the rotor, could reduce the size of the vortices and also increase the outlet flow rate. In this paper, the numerical simulations were conducted by using the Lattice Boltzmann Method and by exploiting the Immersed Boundary method and the Blocking technique in order to overcome the LBM drawbacks. The results show that at the channel height $H^{*}=3.7$, this new model can produce a flow rate of $150 \%$ more than the normal I-Shaped micropumps. Also, one can tune the maximum produced pressure by adjusting the height of this step and micropump with higher channel height can be much more efficient and usable. In addition, by using this new structure for micropump, the designers can also use bigger channel heights which were not efficient in the original design.
\end{abstract}

Keywords: Lattice Boltzmann method; Immersed boundary method; Viscous micropump.

\section{INTRODUCTION}

The scientific advancement in the miniaturization of electromechanical devices necessitates miniaturization of all systems associated with these devices. One of these systems is the fluid transfer system, and it is the most important part, which is a pump.

Micropumps perform the fluid transfer in the micro dimensions. This fluid transfer may be exploited to cool a microchip, a fuel cell, or a micro-reactor, or to transfer, combine, react, or analyze the reactants in a micro total analysis system. Practically, it has been proven that the application of some micropumps, compared to their macro-sized peers, is along with some advantages including reduction in the use of samples and reactants, automating processes, and improving the quality of the experiments.

The surface-to-volume ratio in micro dimensions is much larger than that on the macro scale, hence surface effects will be prominent than the volumetric ones. When the Reynolds number has a small value due to the small flow cross-section or velocity, the viscous forces dominate the inertial ones. Therefore, micropumps using inertial forces as the pumping mechanism have low efficiency. A lot of investigations have been carried out to find new mechanisms that no longer have this problem, leading to the creation of pumping mechanisms including positive displacement using a piezoelectric actuator, phase change, pumping using viscous forces, etc. (Iverson and Garimella 2008).

Among micropumps, positive-displacement micropumps are of higher popularity and are used in various devices, particularly those needing to transfer a specific amount of fluid (for instance, automatic insulin-injection devices). Despite the high popularity, these micropumps require precise fasteners and seals which will make their design complex and therefore, this will make the process of fabrication more difficult.

Electro-hydrodynamic (EHD) pumps are another class of micropumps. In this model, dielectric fluid is exposed to an electric field. This electric field is created by several electrodes located inside the channel, and the fluid is displaced due to induced charges and creates a flow rate inside the channel. Electro-chemical pumps and bubble pumps transfer fluids by utilizing the volume changes from phase transition to displace fluid. Bubble pumps consist of a micro-channel with several independent heaters on its surface. These micro-pumps operate as follows: 
the first heater works enough to form a bubble of fluid's vapor on the surface with the same size as the width of the channel. When the second heater is activated, the bubble expands. Then, by turning on the third heater and turning off the first heater, the vapor bubble starts moving due to the pressure difference in its sides and pushes the fluid forward, toward the micropump outlet. This sequential process continues until the bubble exits the channel, and by that, the process will restart from the beginning. The electrochemical pumps consist of a pair of electrodes located inside a water tank which is connected to a channel filled with fluid. By turning on the pump, the electrodes split the water and create bubbles of oxygen and hydrogen. These bubbles are then transferred to the channel and the fluid inside the channel is driven forwards by these bubbles, creating a flow of fluid.

In viscous micropumps, as the name suggests, the viscosity is utilized as the pumping mechanism. The geometric structure of this class of micropumps includes a cylindrical or disk rotor (a low-height cylinder) placed in a micro-channel in different shapes. In the case of using a disk rotor, the transfer of momentum to the fluid is performed through the base surface of the rotating disk. This category of micropumps can be divided into disk and spiral micropumps. However, when the cylindrical rotor is used, the momentum transfer to the fluid is conducted through the lateral surface of the rotating cylinder. This category can include various types, such as I-shaped, L-shaped, and U-shaped micropumps. All of these micropumps consist of a rotating cylinder asymmetrically located inside the channel. Due to the asymmetry in the rotor position inside the channel, unequal shear forces are created on the top and bottom sides of the rotor during its rotation which causes the fluid to displace. The very simple structure and the lack of need for seals and fasteners are of the advantages of viscous micropumps over other types of micropumps. Moreover, this category of micropumps can be used for all fluids, and energy consumption, compared to other types, is rather low.

I-shaped micropumps were first introduced (Sen et al. 1996). In their experimental study, they observed that the average velocity of the fluid in the micropump outlet was about $10 \%$ of the rotor linear velocity. Besides, they showed that the average velocity and flow rate first increased and then decreased by increasing the channel width at a fixed linear velocity of the rotor. (Sharatchandra et al. 1997) analyzed this micropump numerically and examined the impact of parameters such as the distance of the rotor from the wall, Reynolds number, and pressure difference at the two ends of the micropump on the output flow rate. Moreover, they pointed out in their investigation that two vortices formed above the rotor blocking the flow path and as the channel width increases, vortices will become larger and they start to merge into a single vortex, and as a result, the width of the channel, which flow can pass, will also decrease. In 2004, (Abdelgawad et al. 2004) numerically analyzed the
I-shaped micropump using the Fluent software. Their analysis was carried out unsteadily from $t=0$, i.e. when the rotor was off until the flow reached the steady-state. In this study, they assessed the effect of geometrical parameters on the micropump efficiency, as well as the impact of the Reynolds number, channel width, and the rotor-wall distance on the stability time of the flow and found that the Reynolds number had a higher impact on the stability time that the other parameters. In the same year, (Phutthavong and Hassan 2004)selected different shapes of rotors for an I-shaped micropump and analyzed the flow through the micropump using the Fluent software. One of the findings of their work indicated that the circular rotor was capable of producing more flow rate in comparison to the polygon models. While introducing two novels Lshaped and U-shaped models (Da Silva et al. 2007) obtained optimal values of geometric dimensions for all three models of micropumps. They performed their simulation with the help of the Comsol software.

The aforementioned studies revealed that although in all cases, the effect of geometric parameters, rotor speed, pressure differences, and rotor shape have been investigated, the effect of the channel geometric shape and the vortex formed above the rotor on the flow rate and maximum pressure difference has been neglected. It seems that the position of the vortex or vortices, and eventually the pump flow rate and head can be influenced by changing the flow geometry using additional walls.

In this study, the impact of the additional walls inside the channel on the size of the vortex above the rotor was investigated numerically. From all the investigated cases, only one case could reduce the size of the vortex above the rotor, so that the passage width of the main flow increased and ultimately increased the outlet flow rate. Besides, these new walls enabled us to control the output pressure which micropump produces. In the following, the configuration of these micropumps and the problem geometry are introduced first. The Lattice Boltzmann Method (LBM) and the immersed boundary method (IBM) were used to numerically analyze the flow and define the rotor, respectively. Moreover, the blocking method and the Zou-He model were used to define the additional walls. Finally, the findings will be compared with those reported in previous studies.

\section{Problem Geometry}

The configuration of a simple I-shaped micropump has been depicted in Fig. 1. As shown in this figure, the flow paths are aligned in the inlet and outlet and indicate the letter I (straight line). It should be noted that the naming of the L-shaped and U-shaped micropumps, in which the flow path in the outlet relative to the inlet, changes 90 and 180 degrees, respectively, has been performed in this way. Figure 2 demonstrates the model of an I-shaped micropump with an additional wall (step). The first goal of the selection of this additional wall is to interfere with the flow pattern around the rotor. Furthermore, the 
reason why this configuration has been chosen, in addition to the simplicity of the final geometry, is: based on the definition of the I-shaped micropumps, the flow direction in the inlet and the outlet of the micropump remains the same and fixed. In these figures, D, Lu and $\mathrm{Ld}, \mathrm{H}, \mathrm{Hs}$, and Ls respectively designate the rotor diameter, the inlet and outlet distance to the center of the rotor, the channel height, the step height, and the step length which is used only in the model presented in Fig. 2.

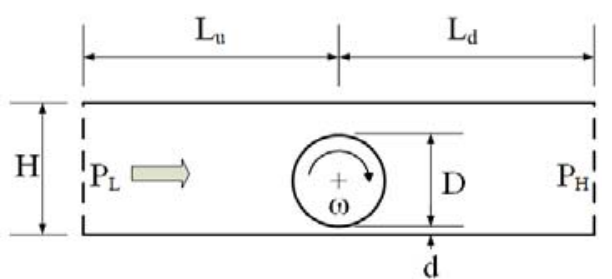

Fig. 1. Simple I-Shaped Viscous Micropump.

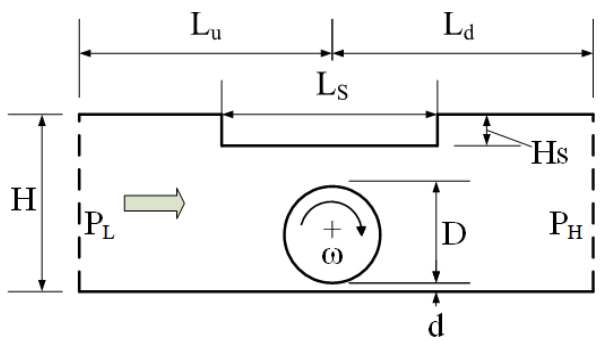

Fig. 2. I-Shaped Viscous Micropump with a step above the rotor.

$d$ is the rotor distance from the bottom wall, with the negative values meaning that part of the rotor is placed within the wall. $\omega$ is the rotor rotational speed in the clockwise direction and $\mathrm{P}_{\mathrm{L}}$ and $\mathrm{P}_{\mathrm{H}}$ are the micropump inlet and outlet pressures, respectively. It is worth noting that the distance of the rotor from the upper wall is not equal to $d$ in Fig. 2, and the vertical position of the upper wall depends only on the value of Hs measured from the upper wall.

By choosing D as the reference length, the geometric dimensions can be nondimensionalized as relations (1).

$$
\begin{aligned}
& x^{*}=\frac{x}{D} \quad y^{*}=\frac{y}{D} \\
& L_{u}^{*}=\frac{L_{u}}{D} \quad H^{*}=\frac{H}{D} \\
& L_{d}^{*}=\frac{L_{d}}{D} \quad d^{*}=\frac{d}{D}
\end{aligned}
$$

Assuming a Newtonian fluid and a laminar, steady, and incompressible flow, the velocity and pressure can be nondimensionalized using (2) and (3), respectively.

$$
U=\frac{\omega D}{2} \quad u^{*}=\frac{u}{U} \quad, \quad v^{*}=\frac{v}{U}
$$

$$
P^{*}=\frac{P}{\rho v^{2} / D^{2}} \quad \Delta P^{*}=P_{H}^{*}-P_{L}^{*}
$$

In the current research, the Reynolds number is written as (4) based on the rotational speed of the rotor.

$$
\operatorname{Re}=\frac{\omega D^{2}}{2 v}
$$

Where $v$ is the fluid kinematic viscosity. The boundary conditions of the walls in the channel and rotor areas are as the non-slip boundary condition and the inlet boundary condition as $P^{*}=P_{L}^{*}=0$, moreover, the outlet boundary condition is as $P^{*}=$ $P_{H}^{*}$, in which $P_{H}^{*}$ can vary from zero to the maximum pressure of that model of the micropump. In addition to these conditions, the derivative of the velocity perpendicular to the boundary at the inlet and outlet boundaries is considered to be zero $\left(\frac{\partial u}{\partial x}=0\right)$. The linear velocity of the rotor is considered to be fixed as $U=$ $\omega D / 2$. The $\mathrm{Lu}$ and $\mathrm{Ld}$ values are assumed to be equal to $8 \mathrm{D}$ so that a fully developed flow can be obtained in the inlet and the outlet and the selected boundary conditions don't harm the simulation. Also, the length of the $\mathrm{L}_{\mathrm{S}}$ has chosen to be $3 \mathrm{D}$.

The power consumption per rotor length can be specified based on the (5).

$$
W^{\prime}=F^{\prime} R \omega
$$

In this relation, the rotor force per unit of length $F^{\prime}$ can be obtained using the following relation.

$$
F^{\prime}=\int_{0}^{2 \pi} \tau_{\text {Wall }} R d \theta
$$

The shear stress on the rotor surface is calculated as (7).

$$
\begin{aligned}
\tau_{\text {Wall }} & =\left.\mu \frac{\partial U_{\theta}}{\partial r}\right|_{r=R} \\
& =\mu \frac{U_{\theta}^{\text {Fluid }}-U^{\text {Rotor }}}{\Delta r}
\end{aligned}
$$

If the rotor starts to rotate at the zero moment, its power consumption will be the maximum, and the power consumption will reduce as the fluid accelerates and will ultimately reach a fixed value. To nondimensionalize the power consumption, the maximum power consumed by the rotor can be selected as the reference power. Assuming $U_{\theta}^{\text {Fluid }}=$ 0 , the maximum rotor power per unit length can be obtained by simplifying the above relations, as (8).

$$
W_{\text {Max }}^{\prime}=\frac{\rho \pi}{D \Delta r} v^{3} R e^{2}
$$

Therefore, by dividing the power consumption by the maximum power consumption, the dimensionless power consumption will be obtained as the following relation. 


$$
W^{*}=\frac{W^{\prime}}{W_{\text {Max }}^{\prime}}
$$

\section{NUMERICAL SOLUTION METHOD}

\subsection{LBM}

The LBM is one of computational fluid dynamics methods in which, instead of solving Navier-Stokes equations, the discrete Boltzmann equation governing the particle distribution function, $f$, is solved. The values of the distribution functions $\mathrm{f}$, in addition to dependence on the discrete locations $x$ and time $t$, they are also dependent on the directions, as each direction is defined by its different particle velocity $C_{i}$. Due to this dependence, the distribution functions $\mathrm{f}$ are represented as $f_{i}$. Selection of the number of directions and the velocity of the particles in each direction leads to different models, with the $\mathrm{D}_{2} \mathrm{Q}_{9}$ model often used in the two-dimensional problems; there are 9 particle velocities in this twodimensional model. In the case of using the Bhatnagar-Gross-Krook (BGK) operator, the LBM can be written as (10). The numerical solution of this relation consists of two stages of the collision and propagation of particles.

$$
\begin{aligned}
f_{i}( & \left.x+c_{i} \Delta t, t+\Delta t\right)-f_{i}(x, t)= \\
= & -\frac{\Delta t}{\tau}\left(f_{i}(x, t)-f_{i}^{e q}(x, t)\right) \\
& +F_{i} \Delta t
\end{aligned}
$$

In this relation $\tau, f_{i}$, and $f_{i}^{e q}$ are the relaxation time constant, discrete force term, and equilibrium distribution functions, respectively.

The value of the equilibrium distribution functions are calculated through relation (11), in which the local density $\rho$ and local velocity $\mathrm{u}$ have been used. In this relation, we have $\vec{u}=u i+v j$ and $\vec{c}=c_{i x} i+$ $c_{i y} j$.

$$
f_{i}^{e q}=w_{i} \rho\left[1+\frac{u \cdot c_{i}}{c_{s}^{2}}+\frac{\left(u \cdot c_{i}\right)^{2}}{2 c_{s}^{4}}-\frac{u \cdot u}{2 c_{s}^{2}}\right]
$$

The particle transfer velocities $c_{i}$ and weighting coefficients $w_{i}$ for this model are given in table 1. In this relation, $c_{s}$ is the sound speed in the fluid and is equal to $c_{s}=\frac{1}{\sqrt{3}} c$, where $\mathrm{c}$ is the particle speed in horizontal and vertical directions in the mesh being $c=\frac{\Delta x}{\Delta y}=1$.

The force term can be written as follows in which $\vec{F}=F_{x} i+F_{y} j$ is the force per unit of volume defined in the macroscopic space.
Table 1 Weighting coefficient and particle velocities for $D_{2} Q_{9}$ Mode

\begin{tabular}{|c|c|c|c|}
\hline$i$ & $\mathrm{w}_{i}$ & $C_{i x}$ & $C_{i y}$ \\
\hline 0 & $4 / 9$ & 0 & 0 \\
\hline 1 & $1 / 9$ & +1 & 0 \\
\hline 2 & $1 / 9$ & 0 & +1 \\
\hline 3 & $1 / 9$ & -1 & 0 \\
\hline 4 & $1 / 9$ & 0 & -1 \\
\hline 5 & $1 / 36$ & +1 & +1 \\
\hline 6 & $1 / 36$ & -1 & +1 \\
\hline 7 & $1 / 36$ & -1 & -1 \\
\hline 8 & $1 / 36$ & +1 & -1 \\
\hline
\end{tabular}

$$
\begin{aligned}
F_{i} & =\left(1-\frac{\Delta t}{2 \tau}\right) \mathrm{w}_{i} \times \\
& \times\left[\frac{c_{i}-u}{c_{s}^{2}}+\frac{\left(c_{i} \cdot u\right) c_{i}}{c_{s}^{4}}\right] \cdot \vec{F}
\end{aligned}
$$

Since the LBM is utilized to analyze flow in the mesoscopic space, the moments of the distribution functions must be used to calculate macroscopic quantities such as density and velocity. For instance, the local density and velocity are obtained through relations (13) and (14), respectively.

$$
\begin{aligned}
& \rho=\sum_{i=0}^{8}\left[f_{i}\right] \\
& \rho \mathrm{u}=\sum_{i=0}^{8}\left[f_{i} \cdot c_{i x}\right]+\frac{\Delta t}{2} F_{x} \\
& \rho \mathrm{v}=\sum_{i=0}^{8}\left[f_{i} \cdot c_{i y}\right]+\frac{\Delta t}{2} F_{y}
\end{aligned}
$$

In the LBM, the pressure is obtained from relation (15), and the relaxation time is related to the kinematic viscosity through relation (16).

$$
\begin{aligned}
& P=c_{s}^{2} \rho \\
& \tau=\frac{v}{c_{s}^{2}}+0.5
\end{aligned}
$$

The BGK collision operator depends only on the parameter $\tau$, which itself is related only to the kinematic viscosity $v$. If the value of $\tau$ is close to 0.5 or it is higher than 1 , then using this operator will cause some problems, for example it can reduce the simulation accuracy, increase simulation time or sometimes it can make the simulation unstable. To solve these problems, the TRT operator has been used for simulation in this study. This operator is dependent on two relaxation times and there is no limitation to determine the relaxation time anymore. Same as BGK operator, one of these relaxation times are obtained from the kinematic viscosity, and another constant is obtained based on the relation 
(17) using a magic number called $\Lambda$. In this simulation, $\Lambda=\frac{1}{12}$ is assumed.

$$
\begin{aligned}
& \tau^{+}= \frac{v}{c_{s}^{2}}+0.5 \\
& \tau^{-}= \frac{\Lambda}{\tau^{+}-0.5}+0.5 \\
& f_{i}\left(x+c_{i} \Delta t, t+\Delta t\right)=f_{i}(x, t) \\
& \quad-\frac{\Delta t}{\tau^{+}}\left(f_{i}^{+}(x, t)-f_{i}^{e q+}(x, t)\right) \\
& \quad-\frac{\Delta t}{\tau^{-}}\left(f_{i}^{-}(x, t)-f_{i}^{e q-}(x, t)\right) \\
& \quad+F_{i} \Delta t \\
& f_{i}^{ \pm}=\frac{f_{i} \pm f_{\bar{i}}}{2} \\
& f_{i}^{e q \pm}=\frac{f_{i}^{e q} \pm f_{\bar{i}}^{e q}}{2}
\end{aligned}
$$

The collision function with the presence of this operator can be observed in relation (18). The procedure of determination of the new distribution functions has been indicated in relation (19); in these relations, $\bar{\imath}$ is the distribution function opposite of the function $i$.

\subsection{Immersed-Boundary Method (IBM)}

Since an orthogonal mesh is often used with uniform distances in rectangular geometries in the LBM, the geometry analysis of the complex flows, such as the flow passing over objects, has always been challenging in this method. For the simulation of the complex flow geometry using the LBM, secondary methods can be used. The immersed boundary method, the ghost fluid method, and the blocking method are among these methods. In this paper, the two methods of blocking and immersed boundary methods have been exploited. It is worth noting that due to the specific features of LBM, the blocking technique can be conveniently used.

The immersed-boundary method is based on the simulation of the flow around the objects (the immersed boundary) introducing the virtual volumetric forces $\mathrm{F}$ in the equations governing the fluid flow field. Different models of this method are based on the procedure of calculation of this force. This method was first proposed by (Peskin 1972) to simulate the flow around the heart valve. The integration of the two methods of LBM and the immersed-boundary method (LB-IBM) was first suggested by (Feng and Michaelides 2004). From that year on, investigations have been conducted to solve this method problems and to improve it. For instance, (Feng and Michaelides 2004) proposed a penalty method to calculate the force, and ( $\mathrm{Wu}$ and Shu 2009) introduced the implicit velocity correction method. This method can simulate an object precisely without the fluid penetrating it, however, due to the implicit nature of this method and the need for solving the equations at all points simultaneously, the simulation of this method is costly and very timeconsuming. In 2011, (Kang and Hassan 2011) removed the problems associated with the velocity correction method and introduced the Multi-DirectForcing method. In this method, construction and inversion of the matrix of coefficients are no longer necessary and the process of solving the equations is performed iteratively. While requiring less time to simulate, this method can produce the results of the implicit velocity correction method accurately.

In the IBM, two series of points are used: Eulerian points and Lagrangian points. In the Eulerian points, in which the location, velocity, and the force is defined respectively as $X_{i, j}=x_{i, j} \vec{\imath}+y_{i, j} \vec{j}, U_{i, j}=$ $u_{i, j} \vec{\imath}+v_{i, j} \vec{\jmath}$, and $F_{i, j}=F x_{i, j} \vec{\imath}+F y_{i, j} \vec{\jmath}$, the main flow equations are solved. It worth mentioning that, $F_{i, j}$ and $U_{i, j}$ replace $\vec{F}$ and $\vec{u}$ in relation (7) and the LBM, respectively.

In the Lagrangian points specifying the boundary of the object and their location, velocity, and force with $X_{k}=x_{k} \vec{\imath}+y_{k} \vec{\jmath}, U_{k}=u_{k} \vec{\imath}+v_{k} \vec{\jmath}$, and $F_{k}=$ $F x_{k} \vec{\imath}+F y_{k} \vec{\jmath}$, respectively, the specific equations of this method, such as the equation for determining the virtual force, are solved. In the case of the rigidity of the object, the distances of these points remain the same and fixed during the solution process. Since Eulerian and Lagrangian points do not necessarily have a direct relationship with each other, a mutual relation must be introduced between these two series of points so that the fluid and boundary of the virtual body feel each other presence. Peskin introduced this relationship using the Dirac delta function so that this function has already become a cornerstone for all IBMs. In this method, first, the velocity of the Lagrangian points is calculated using the velocity interpolation from Eulerian points by the Dirac delta function. Having the velocity at the Lagrangian points, and after calculating the partial forces at these points, these forces are distributed at the Eulerian points to create the required force field to simulate the boundary of the object in the fluid flow. The relation between velocity interpolation and force distribution is written respectively as relations (20) and (21).

$$
\begin{aligned}
& \mathrm{u}_{k}=\sum_{i, j}\left[\mathrm{u}_{i, j} \cdot \Delta\left(X_{i, j}, X_{k}\right)\right] \\
& \mathrm{v}_{k}=\sum_{i, j}\left[\mathrm{v}_{i, j} \cdot \Delta\left(X_{i, j}, X_{k}\right)\right] \\
& \mathrm{Fx}_{i, j}=\sum_{i, j}\left[\mathrm{Fx}_{k} \cdot \Delta\left(X_{i, j}, X_{k}\right)\right] \\
& \mathrm{Fy}_{i, j}=\sum_{i, j}\left[\mathrm{Fy}_{k} \cdot \Delta\left(X_{i, j}, X_{k}\right)\right]
\end{aligned}
$$

In these relations, $\Delta$ is indicative of the Dirac delta function, written as relations (22) and (23) for the two-dimensional problems. 


$$
\begin{aligned}
& \Delta\left(X_{i, j}, X_{k}\right)= \\
& =\phi\left(\left|x_{i, j}-x_{k}\right|\right) \cdot \phi\left(\left|y_{i, j}-y_{k}\right|\right)
\end{aligned}
$$

$$
\begin{aligned}
& \phi(\xi)= \\
& =\left\{\begin{array}{cc}
\frac{1}{4}\left(1+\cos \left(\frac{\pi \xi}{2}\right)\right) & 0 \leq|\xi| \leq 2 \\
0 & 2 \leq|\xi|
\end{array}\right.
\end{aligned}
$$

In the IBM method, the Lagrangian force $F_{k}$ is calculated in different ways, each being the origin of a particular method. In the current study, the MultiDirect-Forcing method proposed by Kang and Hassan has been used to calculate this force, in which the force is calculated according to the following algorithm.

1. Set iteration counter $m=0$

2. Finding the uncorrected velocity from relation (24) and interpolating the Lagrangian velocities from Eulerian points

$$
\begin{aligned}
& \rho \mathrm{u}_{i, j}^{m}=\sum_{\alpha} f_{\alpha} \cdot c_{\alpha x} \\
& \rho \mathrm{v}_{i, j}^{m}=\sum_{\alpha} f_{\alpha} \cdot c_{\alpha y}
\end{aligned}
$$

3. $m=m+1$

4. Calculating the Lagrangian forces from relation (25)

$$
\begin{aligned}
& F x_{k}^{m}=2 \rho \frac{\mathrm{u}_{k}^{d}-\mathrm{u}_{k}^{m-1}}{\Delta t} \\
& F y_{k}^{m}=2 \rho \frac{\mathrm{v}_{k}^{d}-\mathrm{v}_{k}^{m-1}}{\Delta t}
\end{aligned}
$$

5. Spreading the Lagrangian forces to obtain the Eulerian ones.

6. Correct Eulerian velocity with relation (26)

$$
\begin{aligned}
& \mathrm{u}_{i, j}^{m}=\mathrm{u}_{i, j}^{m-1}+\frac{\mathrm{Fx}_{i, j}^{m} \Delta t}{2 \rho} \\
& \mathrm{v}_{i, j}^{m}=\mathrm{v}_{i, j}^{m-1}+\frac{\mathrm{Fy}_{i, j}^{m} \Delta t}{2 \rho}
\end{aligned}
$$

7. Repeat steps 3-7 until $\mathrm{m}$ reaches a pre-defined value $m_{\operatorname{Max}}$

8. Calculate the total correction force with relation (27)

$$
\begin{aligned}
& F x_{i, j}=\sum_{m=1}^{m_{\text {Max }}} F x_{i, j}^{m} \\
& F y_{i, j}=\sum_{m=1}^{m_{\text {Max }}} F y_{i, j}^{m}
\end{aligned}
$$

As previously stated, the blocking technique is another technique helping the LBM to analyze flow; this technique is often used to model fixed rigid bodies in the flow path. In this method, the body is modeled as an object with boundaries coinciding with the mesh points and introduced with the ZouHe boundary condition model, besides, the velocity values of the points inside this block -in the section regarding the computation of macroscopic quantities- are set to be zero. In this paper, as shown in Fig. 6, the step added in the channel of the micropumps have been modeled using this method and the rotor modeled using the IBM.

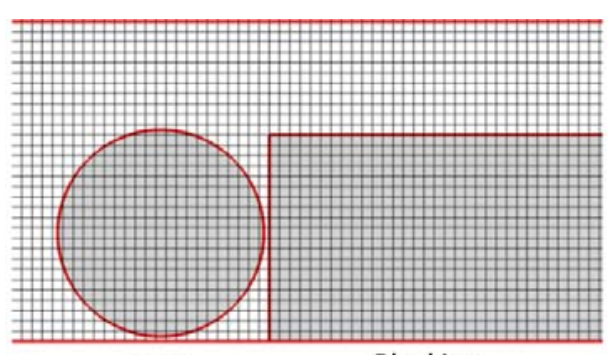

IBM

Blocking

Fig. 3. A sample of generated mesh and Blocking and IB techniques

\section{VALIDATION RESUlTS}

To validate the results, first, the problem of the flow around a cylinder and then the simulation results of an I-shaped micropump are compared with the results of the studies carried out by Abdelgawad et al. 2004 and Da Silva et al. 2007 The solution domain for the problem of the flow around a cylinder can be observed in Fig. 4. The streamlines and vortices behind the cylinder have been demonstrated for a flow with $\operatorname{Re}=40$ in Fig. 5 .

Due to the use of the Multi-Direct-Forcing method, penetration towards inside the fixed cylinder can be witnessed, however, as illustrated in table 2, the vortex length obtained is sufficiently consistent with the results reported by other researchers. Taking into account these results, and as mentioned in reference (Kang and Hassan 2011), it can be claimed that the Multi-Direct-Forcing method (MDF) can produce results similar to those of the Implicit-Direct-Forcing method (IDF), but by spending less time. This time difference in simulation is noticeable, especially for problems with moving or rotating objects. For example, for the current simulation with stationary boundary (for the IDF the process of building and reversing the coefficient matrix was done only once out of the main loop), the simulation for the MDF method required about 1152 seconds while for the IDF, simulation time was about 1030 seconds. In this situation, where the matrix is built only once, the required computation time for the IDF method is about 100 seconds slower than the MDF method. However, if there is a moving or a flexible boundary where you need to rebuild and reverse the matrix for each step of the time, the IDF method will require 
much more time and resources to produce the similar results that the MDF method can produce in a much shorter time.

Once the problem of the flow around a fixed cylinder was investigated, the numerical analysis of the flow in the micropump shown in Fig. 1 was performed and the results were compared with the findings of other studies. Figure 6 illustrates the streamlines around the rotor of this micropump. Given the rotor direction and its proximity to the bottom wall, finally a pure flow has been created to the right with the rotation of the rotor. This is while two relatively large vortex flows have been formed above the rotor.

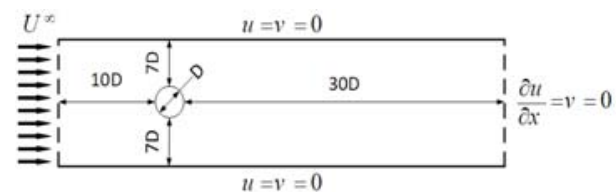

Fig. 4. Geometry of flow past a circular cylinder problem.

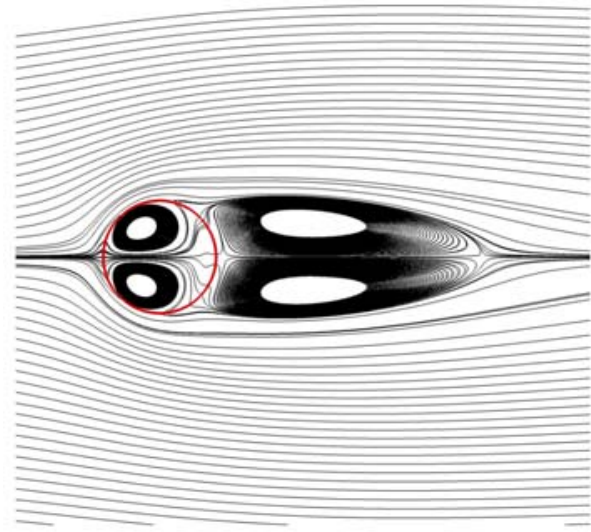

Fig. 5. Geometry of flow past a circular cylinder problem.

Table 2 Comparison between vortex length in present work and other studies

\begin{tabular}{|c|c|c|}
\hline & $\mathrm{Re}=20$ & $\mathrm{Re}=40$ \\
\hline $\begin{array}{c}\text { Wu \& Shu (Wu and } \\
\text { Shu 2009) }\end{array}$ & 1.86 & 4.62 \\
\hline $\begin{array}{c}\text { Shu et al. (Shu, Liu et } \\
\text { al. 2007) }\end{array}$ & 1.8 & 4.4 \\
\hline $\begin{array}{c}\text { Dennis \& Chang } \\
\text { (Dennis and Chang } \\
\text { 1970) }\end{array}$ & 1.88 & 4.69 \\
\hline $\begin{array}{c}\text { Fornberg (Fornberg } \\
\text { 1980) }\end{array}$ & 1.82 & 4.48 \\
\hline $\begin{array}{c}\text { He \& Doolen (He and } \\
\text { Doolen 1997) }\end{array}$ & 1.84 & 4.49 \\
\hline Present Work & 1.84 & 4.57 \\
\hline
\end{tabular}

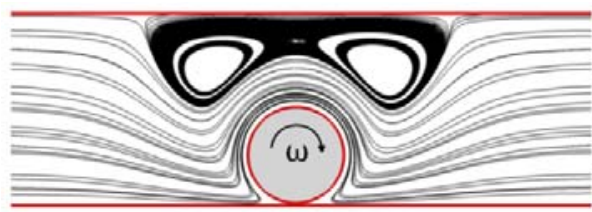

Fig. 6. Streamlines in a simple I-Shaped viscous micropump.

Regarding the interpretation of the cause of the vortices, it should be said that if the length of the channel of this micropump is divided into three zones of the inlet boundary before the vortex flow, the vortex flow zone, and the zone after the vortex flow to the outlet boundary, the negative pressure gradient is the agent for the fluid motion in the first and third zones, and rotation of the moving wall (rotor) in the second zone is the fluid motion factor, As shown in Fig. 7. This is while the presence of a negative pressure gradient in the first and second zones causes a positive pressure gradient in the vortex region. When the fluid moves to the right from the top of the rotor due to the rotor rotation, it cannot continue its way to the right easily in the presence of a positive pressure gradient, and thus diverges to the upper wall. After the collision with the upper wall, part of it deviates to the right and the other part to the left, so that a stagnation point is formed on the upper wall at the right side of the rotor. The backflow near the upper wall cannot continue to the left, as it collides with the main flow and deviates towards the center of the channel to satisfy the principle of mass conservation so that another stagnation point is formed beside the upper wall and before the rotor. The backflow beside the two stagnation points eventually leads to the formation of a vortex region as shown in the figure. However, the flow diverged from the vicinity of the stagnation point before the rotor cannot completely pass over the rotor, and therefore part of it creates a vortex center before the rotor. Accordingly, the diverging flow near the stagnation point after the rotor cannot completely pass near the upper wall, hence creating another vortex center at the right side of the rotor. If the distance between the rotor and the upper wall increase, the flow through this distance is facilitated and the two vortex centers unify and only one vortex is formed above the rotor.

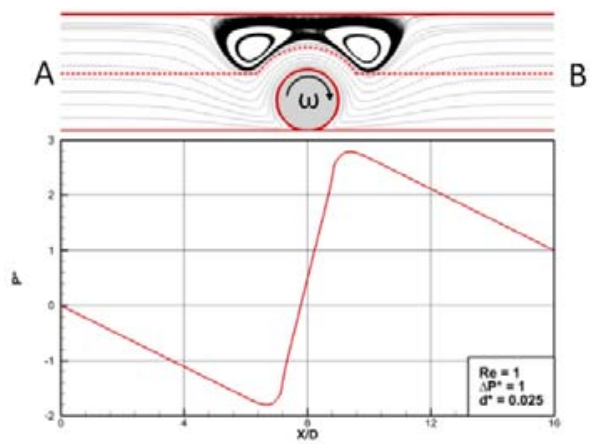

Fig. 7. Pressure change in centerline of an Ishaped viscous micropump. 
Figure 8 depicts the variations in the outlet dimensionless average velocity $u^{*}$ relative to the channel width $H^{*}$, and the average velocity can be obtained through relation (28).

$$
\begin{aligned}
& \bar{u}=\frac{1}{H} \int_{0}^{H} u(y) d y \\
& u^{*}=\frac{\bar{u}}{U}
\end{aligned}
$$

These results have been obtained with values of $R e=0.5, \Delta P^{*}=0.5, \varepsilon=0.9 . \varepsilon$ is a number between 0 and 1 and a controlling parameter for $d$, which are linked by the relation $d=\frac{1}{2}(H-D) \times$ $(1-\varepsilon)$. With a constant $H$ and $D, d$ decreases with an increase in $\varepsilon$. Following these results, the maximum average velocity occurs in a width of $H^{*}=1.53$. It should be noted that in this case, $d^{*}$ also increases with increasing $H^{*}$.

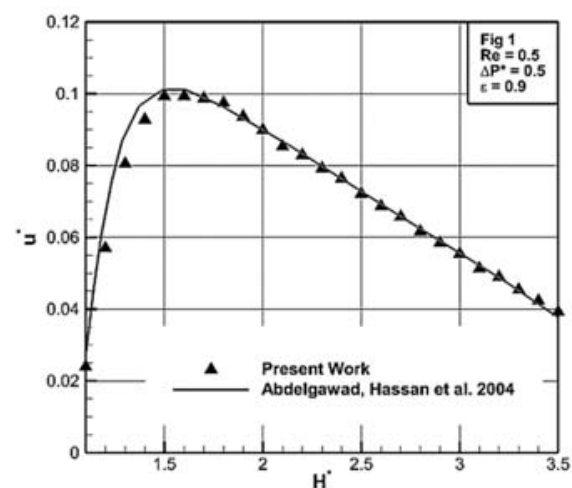

Fig. 8. Comparison of average fluid velocity based on channel height in this work and (Abdelgawad et al. 2004).

Figure 9 illustrates the comparison of variations of the maximum flow rate $\dot{Q}_{\operatorname{Max}}^{*}$ in terms of distance from the wall $d^{*}$ with the corresponding results in the study by Da Silva et al., in which the flow rate is calculated using relation (29).

$$
\dot{Q}^{*}=u^{*} \times H^{*}
$$

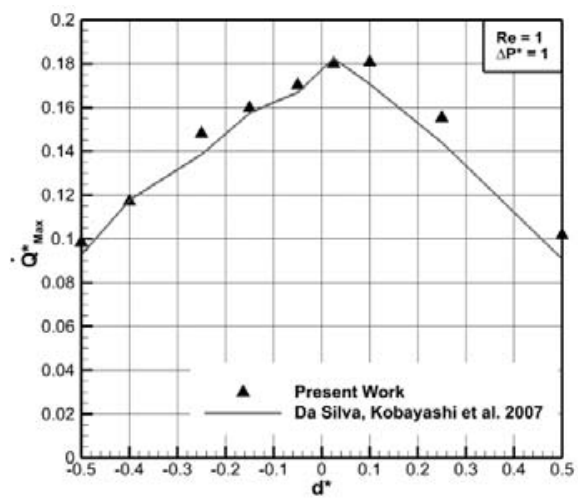

Fig. 9. Comparison of maximum average fluid velocity and maximum flow rate based on rotor distance in this work and (Da Silva et al. 2007).

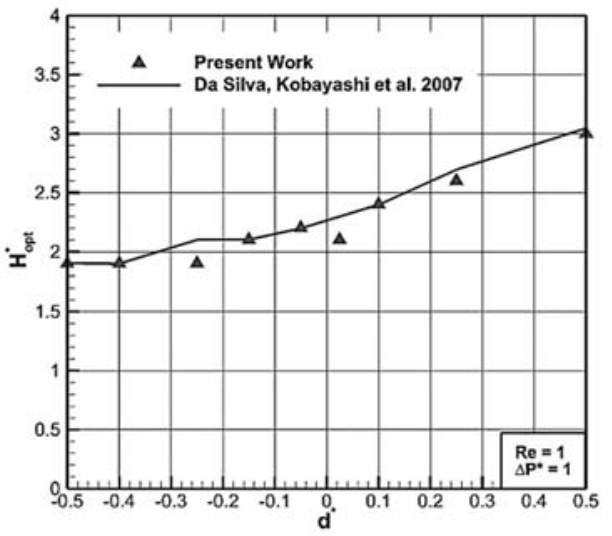

Fig. 10. Comparison of optimum channel height based on rotor distance from bottom wall in this work and (Da Silva et al. 2007).

Based on these results, the flow rate is maximized when $d^{*}=0.025$. It should be noted that in calculating the maximum flow rate, the optimum channel width associated with each $d^{*}$ is always used. It can be seen in Fig. 10 that the optimum channel width increases with increasing $d^{*}$. The optimum channel width is a width in which the outlet flow rate is maximum. In this simulation, the values of the Reynolds number and pressure are assumed to be $R e_{D}=1$ and $\Delta P^{*}=1$, respectively.

As shown in Figs. 8 to 10, the results of the numerical analysis of the present study are highly consistent with the results of other studies.

\section{RESUlts}

The vortex flow above the rotor is one of the main barriers against passing the flow from a viscous micropump, as the width of the main flow decreases with increasing the size of this vortex, ultimately leading to the reduced amount of fluid transferred and the decreased outlet flow rate.

The primary idea in this research was to reduce the negative effect of the vortex flows by adding a wall in the channel. For example, two vortices formed on the rotor of an I-shaped micropump can be observed in Fig. 6, in which $R e=1, \Delta P=1$, and $d^{*}=$ 0.025

In the I-Shaped viscous micropump with a step above the rotor, which is shown in Fig. 2, the step is located above the rotor and its height is measured relative to the upper wall. As demonstrated in Fig. 10 , the added wall can affect the flow behavior at the site of the two stagnation points by controlling the flow and reduce the size of the vortices above the rotor and eventually increase the flow passage width. It is worth mentioning that the height of the added wall is only a small part of the channel width, however, it affects the flow width in such a way that it easily compensates for the decrease in channel width at the rotor site. These changes can be observed in streamlines by comparing Figs. 6 and 11 . 


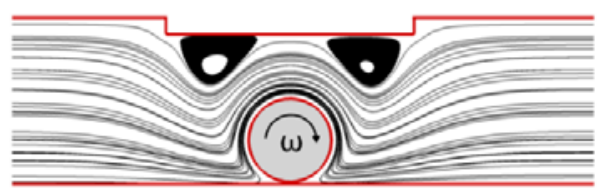

Fig. 11. Streamlines in a viscous micropump of Fig. 2.

If the graph of the effect of the step height on the flow rate is plotted for this micropump, Fig. 12 will be obtained; this figure shows the positive effect of adding the step in the channel on the outlet flow rate, and as shown in this figure, in case of having a certain height, it can increase the outlet flow rate.

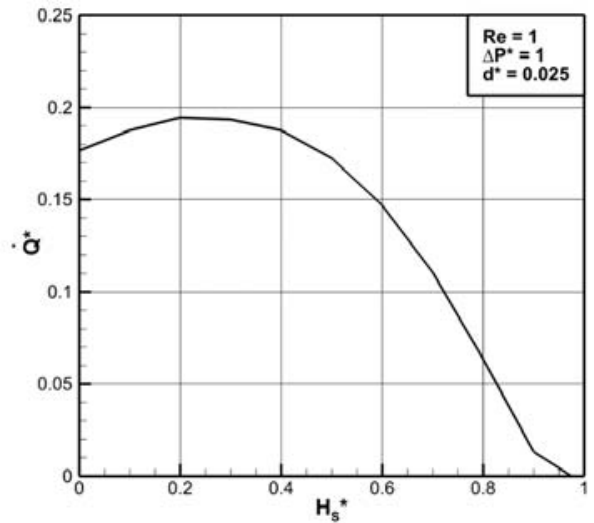

Fig. 12. Effect of height of step in flowrate of viscous micropump of Fig 2.

If the outlet flow rate of this micropump in the stepfree mode and in the optimal mode, in which the flow rate is maximum, is plotted in different channel widths, Fig. 13 will be obtained. As indicated in this figure, in the step-free mode (simple I-shaped micropump), the outlet flow will be maximum, and then will start to decrease due to the reduction of the flow passage width by the vortex flow, however, by adjusting the step in the second micropump, an optimum size can be found for which the formed vortices will have the minimum size and as a result, the flow passage width will increase and this will increase the outlet flow rate. The plot of optimal step height to obtain maximum flow rate based on channel width can be observed in Fig. 14.

According to this graph, it can be seen that up to the channel width of $H^{*}=1.6$, the optimal step height is zero, making the output flow rate be the same in two cases of with and without a step. However, with increasing the channel width, an increase in the step height can be useful to reduce the vortex flow size in addition to increasing the outlet flow rate. This increase in the outlet flow rate is such that in the channel width of $H^{*}=3.7$, the outlet flow rate of the micropump with an optimum height, has an increase of $150 \%$ relative to the outlet flow rate in the stepfree state.

Figure 15 has been obtained by plotting the power consumption diagram of the simple I-shaped micropump and its peer in the presence of an optimum step.

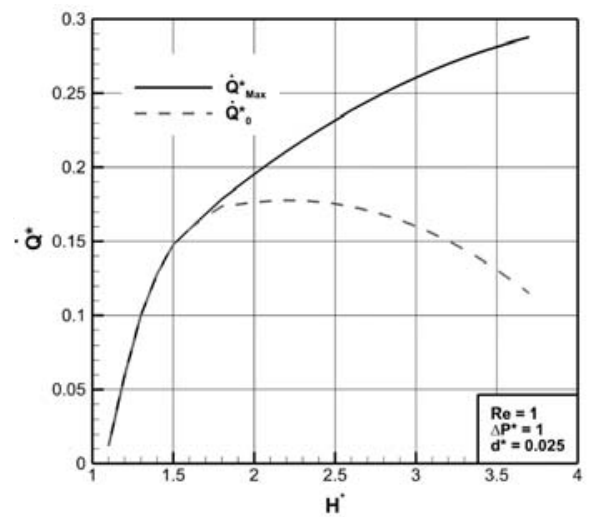

Fig. 13. Comparison of maximum and primary flow rate in a viscous micropump of Fig. 2.

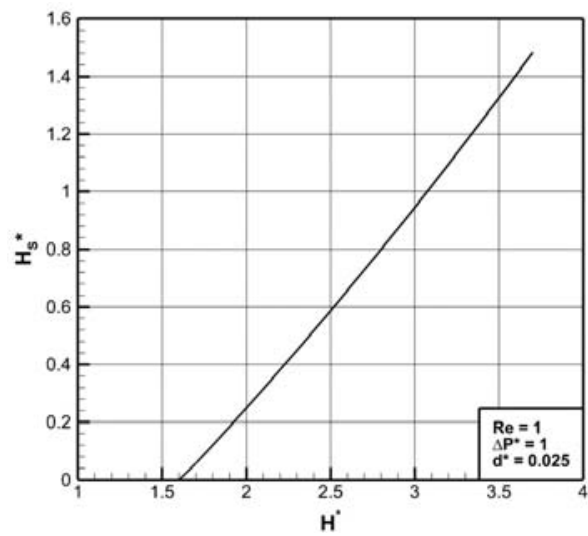

Fig. 14. Optimal step height for maximum flow rate based on channel height in a viscous micropump of Fig. 2.

As can be seen in this figure, surprisingly, the power consumption for both micro-pumps is almost the same, so that they vary by a maximum of $0.15 \%$ in high values of the channel width. Thus, it can be concluded that the step addition does not affect the fluid velocity right around the rotor, and only by controlling the volume of fluid in the vortices (by reducing it), it can increase the flow passage width between the rotor wall and the step wall, so that a higher flow rate is achieved at the same power consumption rate.

If the pressure difference of the two ends of the micropump is equal to zero, i.e. $\Delta P^{*}=0$, the micropump will transfer the maximum flow rate that it can produce. If the graph of the maximum flow rate of the micropumps of Figs. 1 and 2 is plotted, Fig. 16 will be obtained. As can be observed, the maximum flow rate can be increased by adjusting the step height.

A comparison can be observed in Fig. 17 between the maximum pressure differences produced by the micropumps of Figs. 1 and 2. As it is clear, in the simple I-shaped micropump, the maximum pressure difference is maximized with a channel width 
increase, and then the maximum pressure difference has a downward trend. However, in the micropump of Fig. 2, by adjusting the step height, the maximum

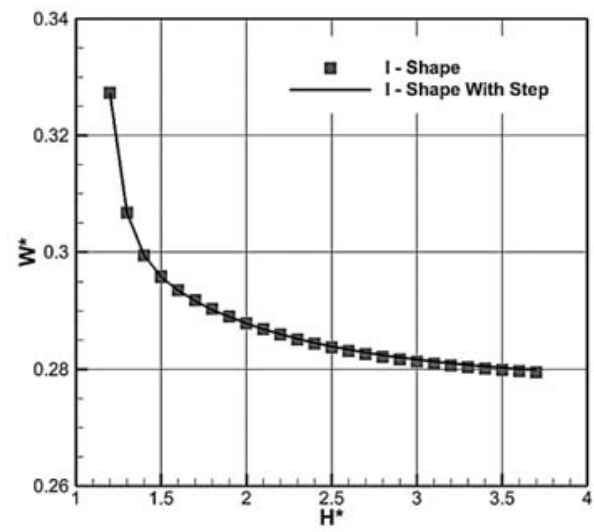

Fig. 15.Comparison between the dimensionless rotor power that viscous micropump Fig. 1 and Fig. 2 need to generate flow.

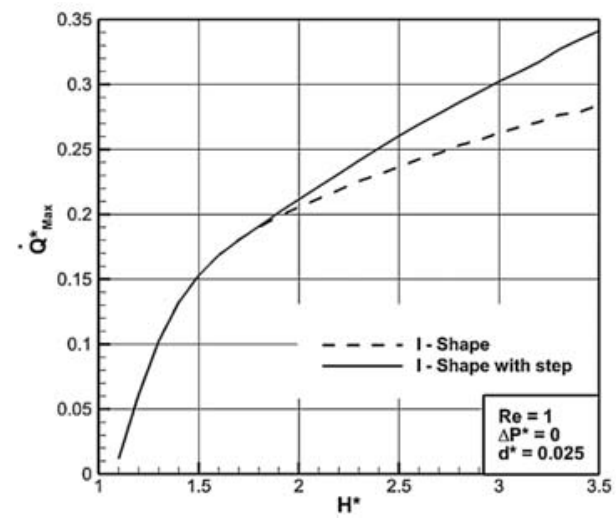

Fig. 16. Comparison of the maximum flow rate that viscous micropumps of Fig 1 and Fig. 2 can produce in the absence of counter pressure.

pressure difference can be produced in all widths of the channel. In Fig. 18, the height of the optimal step height to produce the maximum pressure difference can be observed.

If the free width $H_{F}^{*}=H^{*}-H_{S}^{*}$ at the rotor section (the width between the lower and upper walls at the rotor section in this micropump) is calculated, it can be observed that the free width will be equal to $H_{F}^{*}=1.2$. This means that the free width should be 1.2 to produce the maximum pressure difference. Regarding this result, Fig. 19 has been obtained plotting the maximum pressure difference produced taking into account several channel widths based on the free width; the values of this graph are the same as Fig. 17 for the simple Ishaped micro-pump.

According to this graph, it can be claimed that the maximum pressure difference produced in this micro-pump depends only on the free width value in case of a fixed Reynolds number and the distance from the wall, and this graph can be used to easily specify the maximum pressure difference for all the different channel widths.

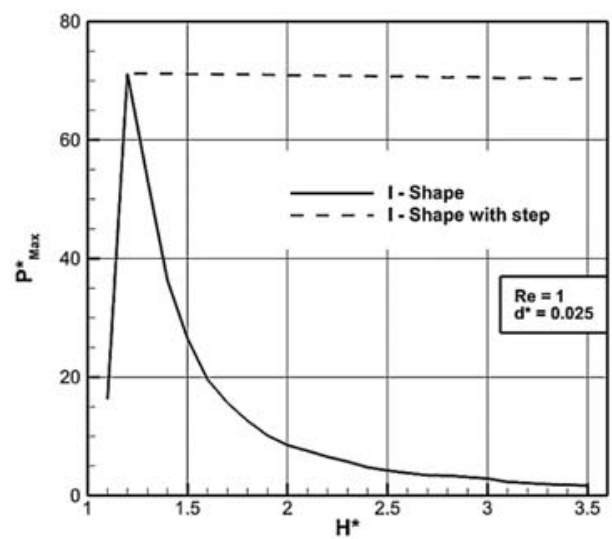

Fig. 17. Comparison of the maximum outlet pressure that viscous micropump of Fig. 1 and

Fig. 2 can produce.

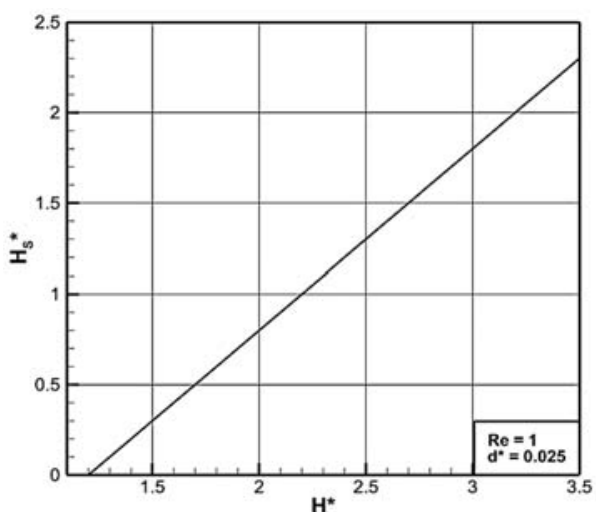

Fig. 18. Optimal step height for maximizing the outlet pressure that a viscous micropump of Fig. 2 can produce based on channel height.

As mentioned before, this can be categorized as a new type of I-Shape viscous micropumps. Therefore, as same as other new pumps, it is necessary to include 2 sets of graphs which are the P-Q graph or "The Pump Performance Curve" and W-Q graph or "The Power Curve". According to the Fig. 20 which is the P-Q curve, this new micropump can withstand more outlet pressure while producing the same flow rate as the normal I-Shape micropump. One can see that at the end of the graph, where there is no outlet pressure, both types of micropump can produce nearly the same flow rate. This can be explained by the absence of the counter-pressure which will reduce the maximum pressure at the right side of the rotor, that micropump will produce to overcome the outlet pressure, and by doing that more fluid can flow at this area which ultimately will reduce the size of the vortex that will develop over the rotor. So, when there is no outlet pressure, the vortex is smaller than there is some outlet pressure, but it still can be 
smaller with adjusting the step height. On the other hand, because it's a small vortex, it has minimum influence over the flow geometry, so the difference between the normal I-Shape and the I-Shape with step micropump flow production is low.

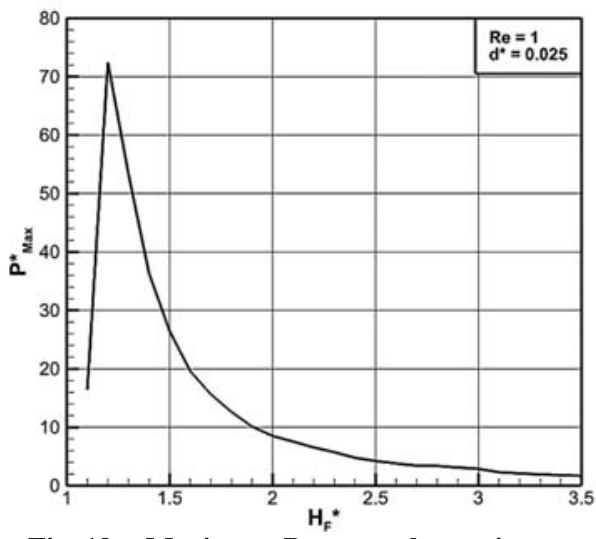

Fig. 19. Maximum Pressure that a viscous micropump of Fig. 2 can produce based on the gap between its bottom and top channel walls at the location of the rotor.

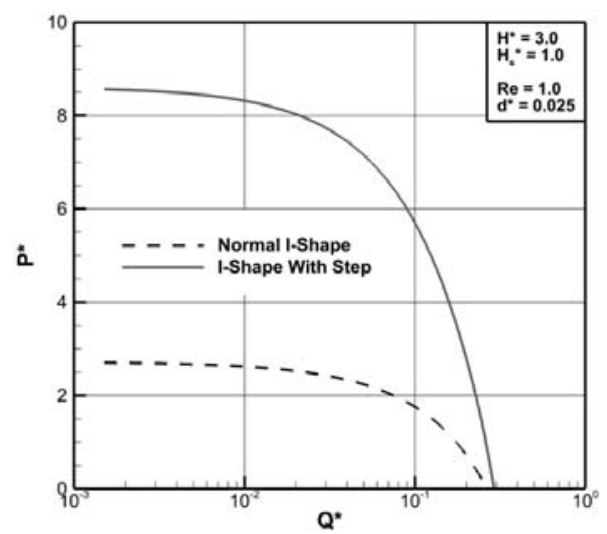

Fig. 20. Comparison between performance curves of Normal I-Shape and I-Shape with step viscous micropump.

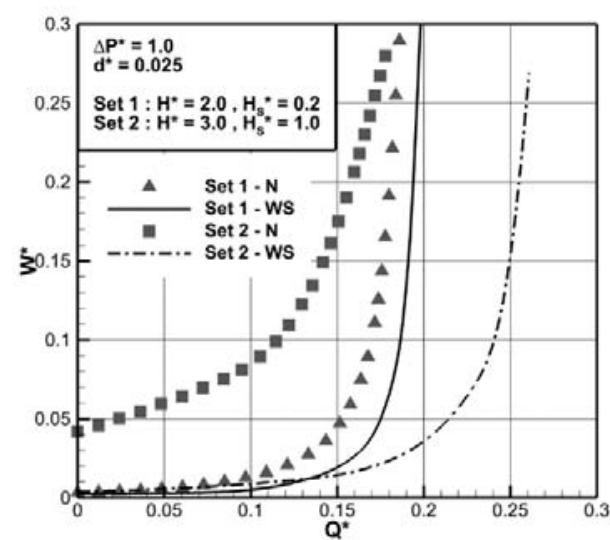

Fig. 21. Comparison between Power requirement curves of Normal I-Shape $(\mathrm{N})$ and I-Shape with step (WS) viscous micropump.
Figure 21 shows the W-Q curve. As it can be observed in this graph, the normal I-Shape micropump requires more power than the I-Shape with step micropump while producing the same flow rate. This can be explained with the help of Fig. 13 and 15 . According to these graphs by increasing the channel height, power consumption by rotor will decrease, and the flow rate in the new viscous micropump increases while in the normal one, this increase will decrease the outlet flow rate. Therefore, in the new micropump, by increasing channel height, one can adjust the flow rate to be the same as the normal one, while the rotor requires less power.

\section{Conclusion}

In this paper, by exploiting the blocking technic and immersed boundary method, two I-Shape viscous micropump -a normal I-Shape micropump and a new I-Shape micropump with an additional step in the micropump channel- were simulated by using the Lattice Boltzmann Method.

In this novel model, by adjusting the step height, the micropump can manipulate the flow in such a way that it can reduce the size of vortices, which are located at the top of the rotor, and as a result, it can increase the main flow pass width and this will increase the outlet flow rate. Besides, by adjusting the step height, one can manipulate the maximum pressure which the micropump can produce. The results show that the extra step doesn't change the fluid velocity which is located in the vicinity of the rotor. Therefore the extra step doesn't change the rotor energy consumption, but by increasing the main flow pass width it can transfer more fluids than before. For example, by setting the channel height $H^{*}=3.7$ and embedding a step with a height of $H_{s}^{*}=1.5$, this novel micropump can produce a $150 \%$ more flow rate than the normal I-Shape viscous micropump.

The results show that by knowing the free width, one can find the maximum pressure which the micropump can produce, or one can adjust this maximum pressure by adjusting the free pass width.

Besides, by using this new structure for micropump, the designers can also use bigger channel heights which were not efficient in the original design.

\section{REFERENCES}

Abdelgawad, M., I. Hassan and N. Esmail (2004). Transient behavior of the viscous micropump. Microscale thermophysical engineering 8(4), 361-381.

Da Silva, A., M. Kobayashi and C. Coimbra (2007). Optimal theoretical design of 2-D microscale viscous pumps for maximum mass flow rate and minimum power consumption. International Journal of Heat and Fluid Flow 28(3), 526-536.

Dennis, S. and G. Z. Chang (1970). Numerical solutions for steady flow past a circular cylinder at Reynolds numbers up to 100. Journal of 
A. Alimoradi and S. Ali Mirbozorgi / JAFM, Vol. 13, No. 6, pp. 1847-1858, 2020.

Fluid Mechanics 42(3), 471-489.

Feng, Z. G. and E. E. Michaelides (2004). The immersed boundary-lattice Boltzmann method for solving fluid-particles interaction problems. Journal of Computational Physics 195(2), 602628.

Fornberg, B. (1980). A numerical study of steady viscous flow past a circular cylinder. Journal of Fluid Mechanics 98(4), 819-855.

He, X. and G. Doolen (1997). Lattice Boltzmann method on curvilinear coordinates system: flow around a circular cylinder. Journal of Computational Physics 134(2), 306-315.

Iverson, B. D. and S. V. Garimella (2008). Recent advances in microscale pumping technologies: a review and evaluation. Microfluidics and nanofluidics 5(2), 145-174.

Kang, S. K. and Y. A. Hassan (2011). A comparative study of direct - forcing immersed boundary lattice Boltzmann methods for stationary complex boundaries. International Journal for Numerical Methods in Fluids 66(9), 1132-1158.

Peskin, C. S. (1972). Flow patterns around heart valves: a numerical method. Journal of computational physics 10(2), 252-271.

Phutthavong, P. and I. Hassan (2004). Transient performance of flow over a rotating object placed eccentrically inside a microchannelnumerical study. Microfluidics and Nanofluidics 1(1), 71-85.

Sen, M., D. Wajerski and M. Gad-el-Hak (1996). A novel pump for MEMS applications. Journal of Fluids Engineering, Transactions of the ASME 118(3), 624-627.

Sharatchandra, M., M. Sen and M. Gad-el-Hak (1997). Navier-Stokes simulations of a novel viscous pump. Journal of Fluids Engineering 119(2), 372-382.

Shu, C., N. Liu and Y. T. Chew (2007). A novel immersed boundary velocity correction-lattice Boltzmann method and its application to simulate flow past a circular cylinder. Journal of Computational Physics 226(2), 1607-1622.

$\mathrm{Wu}$, J. and C. Shu (2009). Implicit velocity correction-based immersed boundary-lattice Boltzmann method and its applications. Journal of Computational Physics 228(6), 1963-1979. 